\title{
Antibiotic resistance trends in paediatric community- acquired first urinary tract infections in the United Arab Emirates
}

H. Narchi' and M.A.M. Al-Hamdani ${ }^{2}$

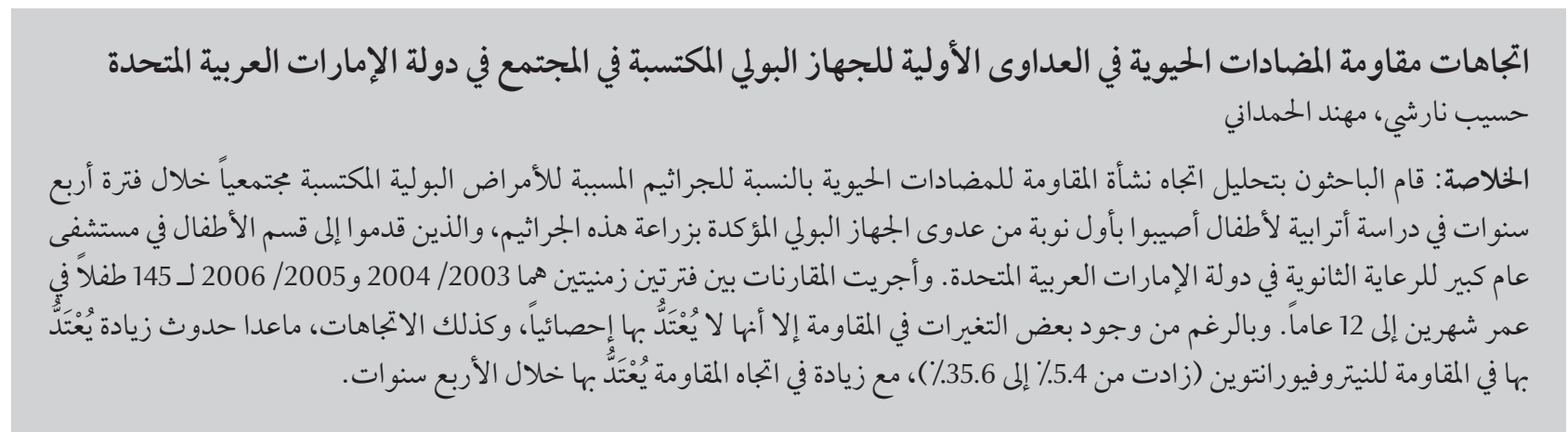

ABSTRACT We analysed the trend of antibiotic resistance of community-acquired uropathogens over a 4-year period in a cohort of children with a first episode of culture-proven urinary tract infection presenting to the department of paediatrics at a large general secondary care hospital in the United Arab Emirates. Comparisons were made between 2 time periods: 2003-04 versus 2005-06 for 145 children aged 2 months to 12 years of age. Although some changes in resistance were observed for some antibiotics between the 2 time periods, the differences were not significant, nor were the trends, except for a significant increase in resistance to nitrofurantoin (from $5.4 \%$ to $35.6 \%$ ), with a significant increasing trend over the 4 years.

Tendances de la résistance aux antibiotiques chez des patients pédiatriques lors d'un premier épisode d'infection urinaire communautaire aux Émirats arabes unis

RÉSUMÉ Pendant quatre ans, nous avons analysé l'évolution de la résistance aux antibiotiques d'agents pathogènes urinaires communautaires dans une cohorte d'enfants connaissant un premier épisode d'infection urinaire confirmé par culture et dirigés vers le service pédiatrique d'un grand hôpital général de niveau secondaire aux Émirats arabes unis. Des comparaisons ont été effectuées entre deux périodes, de 2003 à 2004 et de 2005 à 2006, sur 145 enfants âgés de 2 mois à 12 ans. Bien que l'on ait observé quelques changements sur le plan de la résistance à certains antibiotiques pendant les deux périodes, ni les différences ni les tendances n'étaient significatives, à l'exception d'une nette augmentation de la résistance à la nitrofurantoïne, qui est passée de 5,4 \% à 35,6\% et a montré une importante tendance à la hausse sur la période de quatre ans. 


\section{Introduction}

Urinary tract infections (UTI) are common in the paediatric age group and may be associated with a risk of renal scarring which may lead to hypertension and/or renal damage [1]. To minimize this risk, prompt and appropriate antibiotic therapy is imperative, and is usually initially started empirically before the results of urine culture and bacterial susceptibility are available. Many guidelines exist to recommend the choice of empirical antibiotic treatment, usually based on the knowledge of which organisms are commonly involved and on their antibiotic susceptibility. However, as the latter is known to change over time with the development of antimicrobial resistance, a regular evaluation of the pattern of antibiotic sensitivities is imperative to facilitate the choice of treatment [2-4].

This study assessed the trend of antibiotic resistance of urinary pathogens over a 4-year period in an unselected cohort of children with a first episode of community-acquired UTI, with the aim of improving the choice of initial empirical therapy. We believe that the findings, whether showing a positive or negative or no trend at all in antibiotic resistance, will be of interest to the physician deciding how to treat such infections.

\section{Methods}

The study was undertaken in the department of paediatrics at a large general secondary care hospital in the United Arab Emirates.

\section{Sample size}

Based on prior audit in our institution, the resistance of uropathogens to commonly used antibiotics ranged from $10 \%$ to $70 \%$. As we planned to study several antibiotics with different patterns of resistance, we estimated that for a $95 \%$ confidence level, $5 \%$ significance level and $80 \%$ power to detect an average change of antibiotic resistance from an average of $30 \%-50 \%$ to $60 \%-75 \%$ (or vice versa) between the 2 periods, a minimum a sample size of 49 to a maximum of 66 urine samples in each period (minimum of 98 to a maximum of 132 in total) was needed (Epi-Info statistical software). Assuming that incomplete data may occur in $10 \%$ of these episodes, we decided to analyse a total of 145 UTIs.

\section{Patients}

The inclusion criteria were all children between the age of 2 months and 12 years who had a first episode of cultureproven UTI between 1 January 2003 and 31 December 2006, diagnosed in our institution. The absence of a prior UTI (documented by culture or not), whether in or outside our institution, was specifically ascertained from the child or the parents by history.

The patients were identified from the log books in inpatient wards and outpatient clinics, as well as the microbiology laboratory log book. As per department guidelines, the data collected from all children with UTI (and their parents) is entered on a specifically designed UTI form. This form contained detailed information on the past history, current symptoms and signs, prior relevant investigations or therapy and comorbidity.

We excluded comorbidity or underlying factors shown to be associated with different resistance patterns to antibiotics or a predisposition to recurrent infections which may be associated with a modified resistance profile in view of multiple and/or prolonged courses of antibiotics. Thus the exclusion criteria were: recurrent UTIs (documented or by history, whether in our institution or any other), urine collected by bag specimen, nosocomial UTI, primary or secondary immunosuppression, glucocorticoid therapy, diabetes mellitus, malnutrition, recent urological surgery (within 1 month) or presence of indwelling urinary catheter.

The children were treated with appropriate antibiotics as perantimicrobial sensitivities and underwent appropriate imaging studies as defined in our hospital clinical guidelines.

\section{Urine collection}

The standard departmental protocol for urine collection to diagnose UTI was followed for this study. The mode of urine collection was documented in the microbiology request form and also in the report of urine culture for interpretation purposes.

In children of either sex, under 2 year of age or not yet continent, urine was collected by suprapubic aspiration or catheterization if immediate antibiotic therapy was deemed necessary. Otherwise, or if the parents refused the procedure, 2 samples of urine were collected aseptically by the clean-catch method which, when performed adequately, is as reliable as suprapubic aspiration or catheterization. After cleansing of the genitalia and perineum, the clean catch was collected either by a trained and experienced paediatric nurse, or by the child's mother after appropriate explanation and training by the nurse. UTI was diagnosed by the clean-catch method only if both samples had significant colony counts as defined below. For older and continent children of either sex, urine was collected by the midstream method. The child and/or parents were instructed how to clean the genitalia, retract the foreskin in boys and allow urination to start prior to collecting the midstream in an aseptic manner.

\section{Microbiology}

UTI was defined as the growth of a single pathogen of $>10^{5}$ colony-forming units (CFU)/ $\mathrm{mL}$ of urine collected by clean catch in infants or from midstream in older children with bladder control, or $>10^{3} \mathrm{CFU} / \mathrm{mL}$ if obtained by bladder 
catheterization, or any growth in urine obtained by suprapubic aspiration.

Antimicrobial susceptibility of the isolates was tested by the disk diffusion technique, according to the National Committee for Clinical Laboratory Standards [5]. For the purpose of analysis in this study, organisms with intermediate susceptibility were considered resistant.

\section{Data collection}

The information analysed in the study was derived from the specific UTI form, the case notes and included demographic data, mode of presentation, results of urine cultures and antimicrobial susceptibility. As, for several years, these forms have been prospectively filled on all children with a UTI when they first presented, with excellent completeness of information shown in audits, we believe that their retrospective review for the purpose of this study remains associated with a high level of completeness.

Once the data were entered in a computer file and after checking for completeness and consistency, any information which could allow identification of the participants was removed.

Ethical approval was granted by the Al Ain medical district human research ethics committee (protocol No. 07/116) and no patient consent was required as it was a retrospective observational case notes review.

\section{Analysis}

As the antibiotic resistance pattern was identical between ampicillin and amoxicillin, they were analysed as a single group (aminopenicillins), so too for cefotaxime and ceftriaxone (3rdgeneration cephalosporins).

The data were divided into 2 time periods for analysis: period 1 (years 2003 and 2004) and period 2 (years 2005 and 2006). We used the chi-squared test or Fisher exact test for small numbers (when appropriate) to compare the resistance for each antibiotic between the 2 periods and to look for any association with clinical factors such as age, sex or presence of fever. In addition, we also tested for trend in that change throughout the 4 years of the study. For all the results, statistical significance was defined as $P$-value $<0.05$.

\section{Results}

\section{Clinical descriptive data}

A total of 145 episodes of UTI were diagnosed during the study period. All the information required for the study was present in the UTI form and case notes and there were no relevant missing data. Children under the age of 4 years developed $67.6 \%$ of the episodes (38.6\% under 1 year and $15.2 \%$ between 1 and 2 years of age) and $71.0 \%$ of all infections occurred in females. In period 1 , there were significantly more females $(84.0 \%$ versus $61.0 \%, P<0.001)$ than in period 2 , and there were more younger children aged $<4$ years $(61.9 \%$ versus $71.9 \%$ ) but this was not statistically significant.

Fever was present in $97.2 \%$ of episodes with no difference in the rate between periods 1 and 2, between the sexes or between the 2 age groups ( $<4$ and $4+$ years). Of all the episodes, $94.0 \%$ were managed as inpatients, with a significantly higher proportion in children $<4$ years old (97\% versus $86 \%$, $P<0.05)$, but no difference between the sexes.

Urine was collected by clean catch in $46 \%$, midstream in $41 \%$, suprapubic aspiration in $8 \%$ and bladder catheterization in 5\%. Midstream and catheterization were significantly more frequently performed in females $(P<0.01)$. There was no significant difference in the rates of different collection methods between periods 1 and 2 .
Only 2 children (1.4\%) had received antibiotics (amoxicillin) within 30 days of the diagnosis of UTI; cultures in 1 child were resistant to the same medication while in the other child they still showed sensitivity.

\section{Bacteriology}

Escherichia coli were found in $86.0 \%$ of urine cultures overall ( $87.3 \%$ in period 1 and $85.4 \%$ in period 2), Klebsiella spp. in $6.2 \%(4.7 \%$ in period 1 and $7.3 \%$ in period 2); the remainder were other organisms. There was no significant difference in the proportion of organisms between the 2 periods, nor by age group or sex, nor between first and recurrent episodes (data not shown). Bacteraemia (with the same organism as in the urine) occurred in 2 of the episodes (both with E. coli) and all in children under 1 year of age (1 male and 1 female).

\section{Antibiotic resistance and trends}

In the study of the association between clinical data and antibiotic resistance, only resistance to gentamicin was found to be significantly lower $(P=0.05)$ in the absence of fever $(5 / 133,4 \%)$ compared with febrile episodes $(1 / 4,25 \%)$. Resistance to the other antibiotics was not associated with age, sex or the presence of fever.

The specific resistance pattern to eachorganismis shownin Table 1. There was no significant change in the overall antibiotic resistance between periods 1 and 2, except for a significant 6 -fold increase in resistance to nitrofurantoin (from $5.4 \%$ to $35.6 \%, P<0.001$ ) with a significant increasing trend $(P<0.001)$. There was also a significant decrease in resistance to nalidixic acid $(P<0.05)$ but the test for trend was not significant over the 4 years. Some changes in resistance were observed for some other antibiotics, such as a decrease in resistance to aminopenicillins (from $78.0 \%$ to $72.0 \%$ ), co-amoxiclav (from $62.0 \%$ to $48.6 \%$ ), cefalexin (from $50.0 \%$ to $40.6 \%$ ), and an increase in resistance 


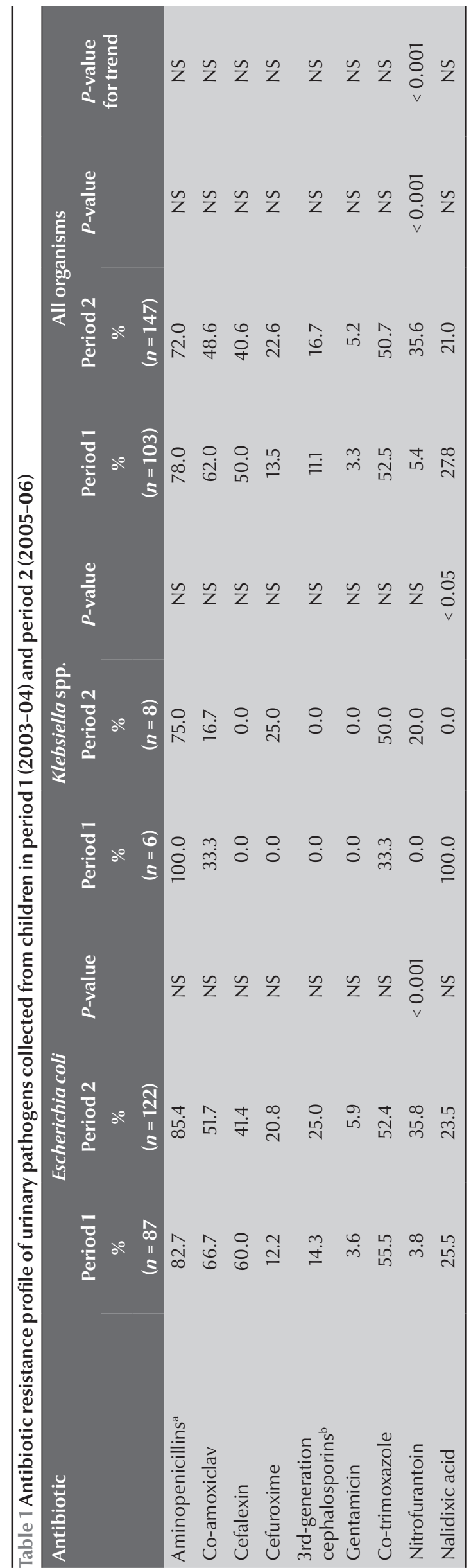

to cefuroxime (from $13.5 \%$ to $22.6 \%$ ), 3rd-generation cephalosporins (from 11.1\% to 16.7\%) and gentamicin (from 3.3\% to 5.2\%); however, the differences were not significant, nor were the trends. The strains resistant to 3rd-generation cephalosporins were sensitive to penicillins, 1st-generation cephalosporins, ceftazidime and aztreonam, and were not considered to be extendedspectrum beta lactamase (ESBL) producers.

\section{Discussion}

As most microbiological surveillance systems lack epidemiological and clinical data to differentiate between first or recurrent episodes of UTI, community- or nosocomially-acquired UTI, complicated or uncomplicated UTI and presence or absence of comorbidity, they may incorrectly estimate the rates of resistance of uropathogens in the community [6]. We aimed to remedy this difficulty by selecting exclusively symptomatic children with a bacteriologically proven communityacquired UTI, excluding all children with asymptomatic bacteriuria or indwelling bladder catheters, all hospitalacquired UTIs and all urine specimens collected by bag where the risk of contamination is very high. The proportion of isolated organisms was similar to other studies, suggesting that the profile of uropathogenic flora in our region is not unusual [7]. Another strength of our study was the completeness of the information available for analysis since we prospectively used standardized data collection forms; this is reflected by the absence of missing data. The study period was restricted to 4 years to coincide with the use of our prospective standardized data collection form for children with UTI (which started in 2003) in order to maximize completeness and accuracy of the data to be analysed. In our community the majority of first UTI in young children are referred to hospital for management and further evaluation; therefore, although the study was carried out in one centre, we believe it to be representative of the general population in this region. However, as we cannot entirely exclude the possibility that some cases of community-acquired UTI may not have been referred, we caution that our results, although representative of hospital data, may not necessarily be generalized to the community.

It is reassuring that resistance to antibiotics of urinary pathogens in community-acquired first UTI has not increased significantly over the 4-year study, with the exception of resistance to nitrofurantoin which has increased 6-fold. As none of these children with a first UTI had ever been on nitrofurantoin prophylaxis, 
long-term exposure to it cannot explain the increase in resistance. In addition, as nitrofurantoin is almost exclusively used in paediatrics for UTI prophylaxis, acquired resistance cannot be blamed on its use in other paediatric conditions. Furthermore, when we analysed the rate of its use as a prophylactic antibiotic for another group of children with recurrent UTI, it was used in less than 5\% of them (the majority were either on co-trimoxazole or a cephalosporin). We therefore could find no support for the theory of an association between antibiotic susceptibility and antibiotic prescribing for the treatment of UTIs, nor can we provide an explanation for the increase in resistance for that antibiotic [8-10]. Reassuringly, we also found a marked decrease in resistance to nalidixic acid in the second period. The reasons are not clear but it could be speculated that the very rare use of that particular antibacterial in our community could explain the increasing sensitivity of uropathogens to it.

The study results differ from a previous study where a tendency for increased resistance to several antibiotics was demonstrated [7]. However, that study compared the resistance pattern between 2 years, 1991 and 1999, and focused exclusively on oral antibiotics, while our study covers a more recent period, which started 4 years later, compared the trend over 4 years instead of 8 , and included parenteral as well as oral antibiotics. It is interesting that the rate of resistance to aminopenicillins, cefalexin, nitrofurantoin and nalidixic acid in that study was fairly similar to our results, except that resistance to co-amoxiclav, cefuroxime and co-trimoxazole were higher in our study and others $[11,12]$.

We found no significant difference in resistance to a specific antibiotic and risk factors such as young age (below 4 years), sex or the presence of fever at presentation, except for resistance to gentamicin which was significantly lower in the absence of fever $(P<0.05)$. This is unlike other studies where female sex was associated with resistance to co-trimoxazole [7]. This may also be explained by the differences in the design of the 2 studies as mentioned above.

In our setting, the most common empirically prescribed antibiotics for UTI (prior to the laboratory sensitivities results) were 1st-, 2 nd- and 3rd-generation cephalosporins, co-amoxiclav and co-trimoxazole. Although it is reassuring that resistance to the 3 generations of cephalosporins and to gentamicin remained below $50 \%$ on average, it was above $50 \%$ to aminopenicillins and co-amoxiclav and around 50\% to co-trimoxazole, similar to other reports $[12,13]$. If these latter antibiotics are empirically selected to treat a UTI instead of cephalosporins, our findings confirm that addition of gentamicin is still required [14]. Empirical therapy with gentamicin, when parenteral treatment is required, is associated with the lowest resistance (less than 5\%). For empirical oral therapy, except for nitrofurantoin and nalidixic acid which still have the lowest resistance (below 50\%), all other choices are associated with resistance above $50 \%$, including co-trimoxazole, as shown in other studies $[14,15]$.
For the treatment of UTI, our findings reinforce the recommendation that cephalosporins (any of the 3 generations) or gentamicin (alone or in combination with aminopenicillins) should be the first choice for empirical therapy $[13,14,16]$. The choice of which of these antibiotics and its route of administration would of course depend on the age of the child, the presence of toxicity or the suspicion of pyelonephritis.

For oral antibiotic prophylaxis, nitrofurantoin and nalidixic acid remain the antibiotics with the lowest resistance rate, while, similar to other studies, we found co-trimoxazole, aminopenicillins, co-amoxiclav and cephalosporins to have more than 50\% resistance [14].

As this study only included children with a first UTI, its findings may not be applicable to recurrent urinary infections, where underlying urinary tract anomalies and the role of antibiotic prophylaxis may lead to different results. This should be studied separately before any recommendations can be made for the choice of empirical antibiotics in children with recurrent UTIs.

\section{Conclusion}

There has been no significant increase in antibiotic resistance to urinary pathogens in community-acquired infections presenting to our hospital between 2003 and 2006, except for nitrofurantoin. There was a decrease in the resistance to nalidixic acid. Continuing surveillance of antibiotic resistance remains essential and requires the incorporation of epidemiological and clinical data into the analysis $[6,17,18]$.

\section{References}

1. Vernon SJ et al. New renal scarring in children who at age 3 and 4 years had had normal scans with dimercaptosuccinic acid: follow up study. British medical journal, 1997, 315:905-8.

2. Hannan M, Cormican M, Flynn J. A comparison of antimicrobial sensitivities of urinary pathogens for the years 1980 and 1990. Irish journal of medical science, 1993, 162:499-501.
3. Dyer IE, Sankary TM, Dawson JA. Antibiotic resistance in bacterial urinary tract infections, 1991 to 1997. Western journal of medicine, 1998, 169:265-8.

4. Shigemura $\mathrm{K}$ et al. Pathogen occurrence and antimicrobial susceptibility of urinary tract infection cases during a 20-year 
period (1983-2002) at a single institution in Japan. Japanese journal of infectious diseases, 2005, 58:303-8.

5. Performance standards for antimicrobial susceptibility testing. Twelfth informational supplement. Wayne, Pennsylvania, $\mathrm{Na}$ tional Committee for Clinical Laboratory Standards, 2002 (Approved Standard. M100-S12).

6. Lopardo G et al. Uropathogen resistance: are laboratory-generated data reliable enough? Journal of chemotherapy (Florence, Italy), 2007, 19:33-7.

7. Prais D et al. Bacterial susceptibility to oral antibiotics in community acquired urinary tract infection. Archives of disease of childhood, 2003, 88:215-8.

8. Tan TY et al. Laboratory antibiotic susceptibility reporting and antibiotic prescribing in general practice. Journal of antimicrobial chemotherapy, 2003, 51:379-84.

9. Christiaens TC, Digranes A, Baerheim A. The relation between sale of antimicrobial drugs and antibiotic resistance in uropathogens in general practice. Scandinavian journal of primary health care, 2002, 20:45-9.

10. Hillier SL et al. How strong is the evidence that antibiotic use is a risk factor for antibiotic-resistant, community-acquired urinary tract infection? Journal of antimicrobial chemotherapy, 2002, 50:241-7.

11. Hernández-Porras M, Salmerón-Arteaga G, Medina-Santillán R. Microbial resistance to antibiotics used to treat urinary tract infections in Mexican children. Proceedings of the Western Pharmacology Society, 2004, 47:120-1.
12. Mehr SS, Powell CV, Curtis N. Cephalosporin resistant urinary tract infections in young children. Journal of paediatrics and child health, 2004, 40:48-52.

13. Lu KC et al. Is combination antimicrobial therapy required for urinary tract infection in children? Journal of microbiology, immunology, and infection, 2003, 36:56-60.

14. Yüksel $\mathrm{S}$ et al. Antibiotic resistance of urinary tract pathogens and evaluation of empirical treatment in Turkish children with urinary tract infections. International journal of antimicrobial agents, 2006, 28:413-6.

15. Goldraich NP, Manfroi A. Febrile urinary tract infection: Escherichia coli susceptibility to oral antimicrobials. Pediatric nephrology (Berlin, Germany), 2002, 17:173-6.

16. Haller M, Brandis M, Berner R. Antibiotic resistance of urinary tract pathogens and rationale for empirical intravenous therapy. Pediatric nephrology (Berlin, Germany), 2004, 19:982-6.

17. Gordon KA, Jones RN. Susceptibility patterns of orally administered antimicrobials among urinary tract infection pathogens from hospitalized patients in North America: comparison report to Europe and Latin America. Results from the SENTRY Antimicrobial Surveillance Program (2000). Diagnostic microbiology and infectious disease, 2003, 45:295-301.

18. Zhanel GG et al. Antibiotic resistance in Escherichia coli outpatient urinary isolates: final results from the North American Urinary Tract Infection Collaborative Alliance (NAUTICA). International journal of antimicrobial agents, 2006, 27:468-75.

\section{Handbook: Good Laboratory Practice (GLP). Second Edition}

Good laboratory practices (GLP) are the recognized rules governing the conduct of non-clinical safety studies. They ensure the quality, integrity and reliability of the study data.

This handbook is designed as an aid for those countries wishing to upgrade their laboratories to GLP status. Based on the Organisation for Economic Cooperation and Development (OECD) principles of GLP, the aim of the handbook is to provide laboratories and trainers in disease-endemic countries with the necessary technical information for implementing GLP programmes.

The material in the handbook is presented in a clear and informative way. The introduction reviews the need for quality standards in drug research and development, and gives a history of GLP. Chapter 2 covers GLP training, and chapter 3, the stepwise introduction of GLP. The OECD principles of GLP and compliance monitoring are reprinted, with kind permission, in the annex section.

Further information about this and other WHO publications can be found at: http:/ / www.who.int/bookorders / anglais $/$ home 1 .jsp? sesslan=1 